\title{
No evidence that bonding is needed for amalgam restorations
}

\author{
Abstracted from \\ Agnihotry A, Fedorowicz Z, Nasser M. \\ Adhesively bonded versus non-bonded amalgam restorations for dental caries. \\ Cochrane Database Syst Rev 2016; 3: Art. No. CD007517. DOI: 10.1002/14651858.CD007517.pub3. \\ Address for correspondence: Luisa Fernandez Mauleffinch, Managing Editor, Cochrane Oral Health Group, \\ School of Dentistry, The University of Manchester, JR Moore Building, Oxford Road, Manchester, M13 9PL, UK. \\ E-mail: luisa.fernandez@manchester.ac.uk
}

\section{Question: Is there any difference in service performance and longevity in the use of adhesive bonding compared to no use of bonding in dental amalgam restorations?}

Data sources Relevant databases were searched for the review such as the Cochrane Oral Health Group Trials Register, Medline, EMBASE, clinical trials.gov, WHO International Clinical Trials Registry Platform. No restrictions on the language or date of publication were applied to the searches. Other resources for searching for possible trials included databases of ongoing clinical trials and reference lists of relevant articles. Key investigators were contacted. No hand searching was performed.

Study selection Randomised controlled trials comparing adhesively bonded versus non-bonded class I and II amalgam restorations in permanent molars and premolars.

Data extraction and synthesis Two reviewers independently screened papers and extracted data.

Results One trial with 31 patients who received 113 restorations was included. At two years, 50 out of 53 restorations in the nonbonded group survived and 55 of 60 restorations survived with five unaccounted for at follow-up. Post insertion sensitivity was not statistically significant at baseline and for the two-year follow-up. No fractures or differences in the marginal adaptation were reported. Conclusions There is no evidence either to claim or to refute a difference in survival between bonded and non-bonded amalgam restorations. In view of the lack of evidence on the additional benefit of adhesively bonding amalgam in comparison with non-bonded amalgam, it is important that clinicians be mindful of the additional costs that may be incurred.

\section{Commentary}

Amalgam is still the material of choice for posterior restorations in many places and for many practitioners despite the availability of more aesthetic restorations and despite the efforts for the use of amalgam to be removed from general practice. ${ }^{1}$

The continuous use of amalgam is supported by the material properties and the technique needed for application that is still less sensitive than composite restorations for posterior teeth.

The appropriate methodology was used in the Cochrane review. The proper study design, randomised clinical trials, were searched to answer the clinical question.

Sufficient databases were searched and the body of evidence was properly analysed from only one clinical trial that was included in the final analysis.

The overall body of evidence for the outcome was analysed to be low and the authors concluded that there is no evidence that using bonding materials makes a difference in the longevity and in the outcome of sensitivity in amalgam restorations.

As practitioners we certainly have to agree with the authors' conclusions. Amalgam is still a suitable material for dental restorations $^{2}$ despite new evidence and claims about the possible adverse events associated with the material. ${ }^{3}$

However, research including the use of bonding may not be needed. The use of bonding agents has no beneficial or detrimental effect on the clinical performance of amalgam restoration, but is adding an extra step and cost to the restoration. The only advantage seems that, by using bonding, less tooth structure needs to be removed for the mechanical retention of the final restoration.

If you still prefer the use of amalgam for posterior restorations, you may not need to use bonding agents if you're not using them yet.

James R Keenan and Analia Veitz-Keenan NYU College of Dentistry, New York, USA

This paper is based on a Cochrane Review published in the Cochrane Library 2016, issue 3 (see www.thecochranelibrary.com for information). Cochrane Reviews are regularly updated as new evidence emerges and in response to feedback, and the Cochrane Library should be consulted for the most recent version of the review.
1. Kopperud SE, Staxrud F, Espelid I, Tveit AB. The Post-Amalgam Era: Norwegian Dentists' Experiences with Composite Resins and Repair of Defective Amalgam Restorations. Int J Environ Res Public Health 2016; 13: 441. doi: 10.3390/ijerph13040441.

2. Rasines Alcaraz MG, Veitz-Keenan A, Sahrmann P, Schmidlin PR, Davis D, IheozorEjiofor Z. Direct composite resin fillings versus amalgam fillings for permanent or adult posterior teeth. Cochrane Database Syst Rev 2014; 3: CD005620. doi: 10.1002/14651858.CD005620.pub2.

3. Kern JK, Geier DA, Bjørklund G, et al. Evidence supporting a link between dental amalgams and chronic illness, fatigue, depression, anxiety, and suicide. Neuro Endocrinol Lett 2014; 35: 537-552. 